\title{
Performance-Related Sustained and Anticipatory Activity in Human Medial Temporal Lobe during Delayed Match-to-Sample
}

\author{
Rosanna K. Olsen, ${ }^{1}$ Elizabeth A. Nichols, ${ }^{1}$ Janice Chen, ${ }^{1}$ Jack F. Hunt, ${ }^{1}$ Gary H. Glover, ${ }^{2,3}$ John D. E. Gabrieli, ${ }^{4}$ \\ and Anthony D. Wagner ${ }^{1,3}$ \\ Departments of ${ }^{\text {Psychology and }}{ }^{2}$ Radiology, and ${ }^{3}$ Neurosciences Program, Stanford University, Stanford, California 94305, and ${ }^{4}$ Department of Brain \\ and Cognitive Sciences, Massachusetts Institute of Technology, Cambridge, Massachusetts 02139
}

The medial temporal lobe (MTL) — hippocampus and surrounding perirhinal, parahippocampal, and entorhinal cortical areas_- has long been known to be critical for long-term memory for events. Recent functional neuroimaging and neuropsychological data in humans performing short-delay tasks suggest that the MTL also contributes to performance even when retention intervals are brief, and singleunit data in rodents reveal sustained, performance-related delay activity in the MTL during delayed-non-match-to-sample tasks. The current study used functional magnetic resonance imaging to examine the relationship between activation in human MTL subregions and performance during a delayed-match-to-sample task with repeated (non-trial-unique) stimuli. On critical trials, the presentation of two faces was followed by a $30 \mathrm{~s}$ delay period, after which participants performed two-alternative forced-choice recognition. Functional magnetic resonance imaging revealed significant delay period activity in anterior hippocampus, entorhinal cortex, and perirhinal cortex over the $30 \mathrm{~s}$ retention interval, with the magnitude of activity being significantly higher on subsequently correct compared with subsequently incorrect trials. In contrast, posterior hippocampus, parahippocampal cortex, and fusiform gyrus activity linearly increased across the $30 \mathrm{~s}$ delay, suggesting an anticipatory response, and activity in parahippocampal cortex and hippocampus was greater during the probe period on correct compared with incorrect trials. These results indicate that at least two patterns of MTL delay period activation - sustained and anticipatory - are present during performance of short-delay recognition memory tasks, providing novel evidence that multiple processes govern task performance. Implications for understanding the role of the hippocampus and surrounding MTL cortical areas in recognition memory after short delays are discussed.

\section{Introduction}

Although the necessity of the medial temporal lobe (MTL) for declarative memory performance after long delays is well established (Cave and Squire, 1992; Squire, 1992), recent data indicate that MTL damage can impair recognition memory for complex visual stimuli (e.g., faces, objects, and scenes) or the relationships between stimuli, even when memory is tested after delays as brief as 1-8 s (Hannula et al., 2006; Nichols et al., 2006; Olson et al., 2006a,b; Hartley et al., 2007; Piekema et al., 2007). Such deficits have been interpreted as evidence that working memory mechanisms (e.g., active maintenance) are MTL dependent (Ranganath and Blumenfeld, 2005; Hasselmo and Stern, 2006), or as evidence that the MTL mediates short-delay performance when working memory is insufficient (Warrington and Taylor, 1973; Shrager et al., 2008).

Received May 12, 2009; revised July 6, 2009; accepted Aug. 5, 2009.

This work was supported by National Institute of Mental Health Grant 5R01-MH076932, a National Science Foundation Graduate Fellowship, the National Alliance for Research on Schizophrenia and Depression, and The Alfred P. Sloan Foundation. We thank C. Brock Kirwan and Can Ceritoglu for their advice on ROI alignment analysis methodology and Jesse Rissman for helpful discussions.

Correspondence should be addressed to Rosanna K. Olsen, Department of Psychology, Jordan Hall, Building 420, Stanford University, 650 Serra Mall, Stanford, CA 94305. E-mail: rkolsen@stanford.edu.

DOI:10.1523/JNEUROSCI.2245-09.2009

Copyright $\odot 2009$ Society for Neuroscience 0270-6474/09/2911880-11\$15.00/0
Functional magnetic resonance imaging (fMRI) data also implicate the MTL in short-delay recognition memory (Ranganath and D'Esposito, 2001; Schon et al., 2004; Ranganath et al., 2005; Nichols et al., 2006; Piekema et al., 2006; Axmacher et al., 2007). For example, Ranganath et al. (2005) observed MTL delay period activity when subjects performed delayed-match-tosample (DMS) with a 7-13 s retention interval. However, although this activity predicted postscanning recognition performance-suggesting a role in episodic encoding that supports long-delay retrieval-ceiling-level DMS performance prevented assessment of whether MTL delay period activity also relates to immediate recognition. Moreover, because this and most other fMRI studies revealing delay period MTL activity used (1) relatively short delay periods and (2) trial-unique stimuli that may differentially foster episodic encoding (Ranganath and D’Esposito, 2001; Schon et al., 2004) (cf. Piekema et al., 2006), there remains uncertainty about the functional significance of MTL "delay period" activity - it could reflect true delay period blood oxygen level-dependent (BOLD) signal or carryover of study period BOLD responses, and it may be specific to novelty encoding processes that support long-term memory.

Within the MTL, short-delay recognition memory may be differentially subserved by distinct subregions. Persistent activity 


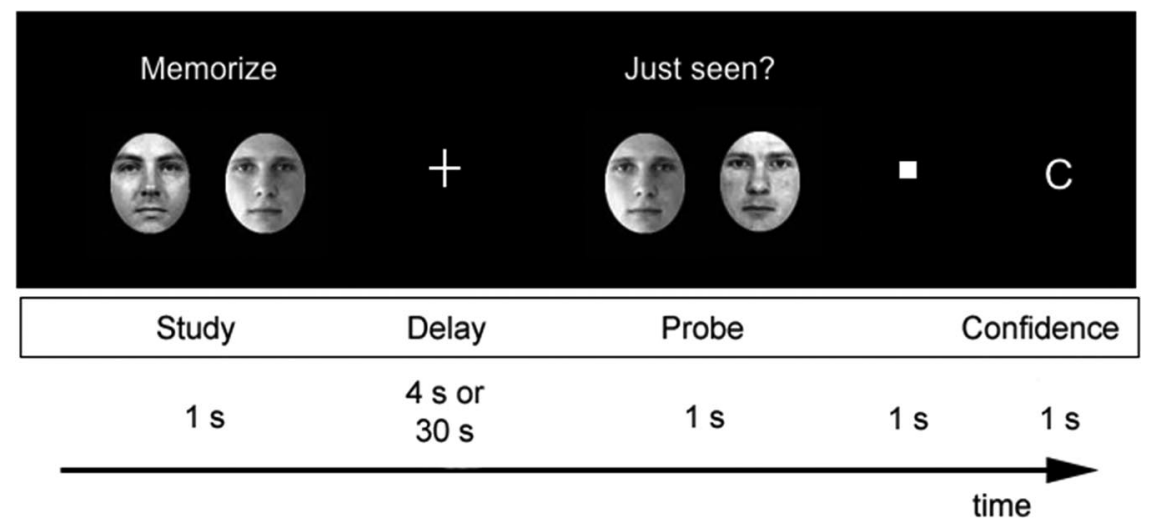

Figure 1. Diagram of task design. Two faces were simultaneously shown during the study period for $1 \mathrm{~s}$, followed by either a $4 \mathrm{~s}$ or a 30 s delay period. Subsequently, two test faces were shown simultaneously for $1 \mathrm{~s}$ (participants had $2 \mathrm{~s}$ to respond), and then a " $C$ " was displayed for $1 \mathrm{~s}$ cuing participants to rate their memory confidence.

in entorhinal cortex is putatively driven by an intrinsically generated mechanism and is posited to underlie active maintenance of goal-relevant stimuli (Egorov et al., 2002; Fransen et al., 2002). Consistent with this hypothesis, time-dependent recognition deficits emerge after selective entorhinal lesion (Van Cauter et al., 2008). Moreover, in rodent hippocampus, specific subfields show dynamic performance-related patterns of sustained delay period activity during delayed-non-match-to-sample over a $30 \mathrm{~s}$ delay (Deadwyler and Hampson, 2004). Characterization of delay period responses in specific human MTL cortical and hippocampal regions requires higher spatial resolution fMRI methods than previously implemented.

In the present study, fMRI of DMS task performance was used to address three central questions: (1) Does the human MTL demonstrate sustained activity during DMS performance that unambiguously reflects delay period processes? (2) Which MTL substructures demonstrate delay period responses, and does the temporal profile of this activity differ across regions? (3) Does MTL delay period activity predict immediate subsequent DMS performance? Using familiarized (non-trial-unique) stimuli and a $30 \mathrm{~s}$ delay, we examined the temporal profile of delay period activation in each MTL subregion, and the relationship between study, delay, and probe period activation and DMS performance.

\section{Materials and Methods}

Subjects. Twenty-five right-handed, native-English speakers (10 females; ages, 18-32 years) were recruited from the Stanford University community and surrounding areas. Subjects were paid $\$ 70$ for their participation. Data from 5 of the original 25 subjects were excluded from analysis ( 3 because of poor behavioral performance and 2 because of functional data artifacts). Informed consent was obtained in a manner approved by the institutional review board at Stanford University.

Behavioral procedures. Subjects performed a DMS task using familiarized face stimuli. Each trial consisted of four phases: study, delay, probe, and confidence judgment (Fig. 1). In the study phase, two faces and the prompt "Memorize" were simultaneously presented for $1 \mathrm{~s}$, and participants were instructed to try to remember the faces. Two faces were used (instead of one face) to increase task difficulty, thus yielding a sufficient number of correct and incorrect trials to permit performance-related fMRI analyses (see below). In the delay phase, a fixation cross was presented for either $4 \mathrm{~s}$ ( $50 \%$ of trials) or $30 \mathrm{~s}$ ( $50 \%$ of trials). To encourage active maintenance, participants were instructed to keep both faces "in mind" over the delay period so that they would be ready to respond when the probe faces appeared. Trials were intermixed, ensuring that participants were unaware of the delay length for a given trial before experiencing the delay. In the probe phase, participants made a two- alternative forced-choice recognition decision. Specifically, two faces and the prompt "Just seen?" were presented for $1 \mathrm{~s}$ followed by a small square for $1 \mathrm{~s}$, and participants were instructed to indicate which of the two probes had been presented in the study phase on that trial. During this $2 \mathrm{~s}$ period, participants pressed a button with their right index finger if the studied face was on the left (50\% of trials) or with their right middle finger if the studied face was on the right $(50 \%$ of trials). Finally, in the confidence judgment phase, the letter " $C$ " was presented for $1 \mathrm{~s}$, which prompted participants to press one of two buttons indicating whether they were confident (index finger) or not confident (middle finger) about their probe phase response. A $1 \mathrm{~s}$ centrally presented asterisk preceded each trial to alert participants that a new trial was beginning, and a $9 \mathrm{~s}$ intertrial interval followed each trial during which

subjects viewed a fixation cross.

For the DMS task, stimuli were drawn from a set of 30 faces, such that stimuli were not trial unique (i.e., the same 30 faces appeared repeatedly during the DMS experiment). To minimize reliance on item familiarity as a basis for recognition decisions, participants were familiarized with the 30 faces used in the DMS task before the experiment, encountering each face 10 times during a target detection task. Specifically, before scanning, participants viewed a stream of 330 face trials (the 30 DMS faces 10 times each and 2 "targets" 15 times each) and made a button press whenever the face was one of the targets. On each trial, a face was presented ( $1 \mathrm{~s})$ followed by a fixation $(0.2 \mathrm{~s})$. Target and nontarget faces were intermixed in a pseudorandom order, with the constraint that each of the nontarget faces appear once during every 30 nontarget trials. All stimuli were grayscale images of young, white, male, nonfamous faces with hair cropped out (Fig. 1). We note that, although this pre-DMS familiarization procedure, along using non-trial-unique stimuli, was designed to minimize participants' reliance on differential familiarity as a basis for recognition decisions, it nevertheless remains possible that relative familiarity differences between the target and foil during the DMS task could contribute to performance.

Before entering the scanner, participants received brief practice on the DMS task. Subsequently, the DMS task was performed during 12 functional scans, each consisting of five $4 \mathrm{~s}$ delay trials and five $30 \mathrm{~s}$ delay trials intermixed in a pseudorandom manner. Across the entire experiment, each of the 30 faces was presented 16 times: 8 times in the study phase (appearing equally often in $4 \mathrm{~s}$ trials and in $30 \mathrm{~s}$ trials, and appearing equally often on the left and on the right side) and 8 times in the probe phase (appearing equally often as the studied stimulus and as the unstudied stimulus, and equally often on the left and right). Together using familiarized, non-trial-unique stimuli, inclusion of the $4 \mathrm{~s}$ delay trials was designed to encourage participants to actively maintain the study faces during the delay period. On $4 \mathrm{~s}$ delay trials, the delay period fMRI response cannot be unambiguously deconvolved from the study period and probe period responses, and thus we restricted our analyses to the 30 s delay trials (see below, fMRI procedures).

For all tasks, stimuli were presented and responses were recorded using Matlab 7 (Mathworks) using the Psychophysics Toolbox extensions (Brainard, 1997; Pelli, 1997). Responses during the target detection task were made using an Apple Powerbook laptop and responses during the DMS task were made with the right hand using a scanner-compatible button box.

To avoid gradient coil overheating, intermittent breaks were required between the scanned DMS task runs. To keep participants alert throughout the scanning session, these break periods were filled with a verbal one-back task in which participants had to determine whether the pseudoword on the screen was the same as the pseudoword presented in the trial immediately before. Each break period contained 49 filler trials, 
and lasted $2 \mathrm{~min}$ and $13 \mathrm{~s}$; across participants, a total of six to eight break periods was required during the scanning session.

fMRI procedures. Imaging data were acquired on a 3.0 T Signa wholebody MRI system (GE Medical Systems) with a homemade quadrature transmit/receive head coil. Head movement was minimized using a "bite bar" and additional foam padding. Before functional imaging, highresolution, T2-weighted, spin-echo structural images [repetition time $(\mathrm{TR})=3000 \mathrm{~ms}$; echo time $(\mathrm{TE})=68 \mathrm{~ms} ; 0.43 \times 0.43 \mathrm{~mm}$ in-plane resolution] were acquired in 223 -mm-thick slices perpendicular to the main axis of the hippocampus allowing for the segmentation of hippocampal subfields (dentate gyrus/ $\mathrm{CA}_{2 / 3}, \mathrm{CA}_{1}$, and subiculum) and MTL cortical areas (perirhinal, parahippocampal, and entorhinal cortices).

A total of 972 functional volumes were acquired for each participant using a $\mathrm{T}^{*}{ }^{*}$-sensitive gradient echo spiral in/out pulse sequence (Glover and Law, 2001) with the same slice locations as the structural images (TR, $4000 \mathrm{~ms}$; TE, $34 \mathrm{~ms}$; flip angle, $90^{\circ}$; two shots; field of view, $22 \mathrm{~cm}$ ). Functional data were acquired a higher resolution than is usually acquired during imaging of human MTL: $1.89 \times 1.89 \times 3.00 \mathrm{~mm}$. A high-order shimming procedure, based on spiral acquisitions, was used to reduce $B_{0}$ heterogeneity (Kim et al., 2002). Critically, spiral in/out methods are optimized to increase signal-to-noise and BOLD contrastto-noise ratio in uniform brain regions while reducing signal loss in regions compromised by susceptibility-induced field gradients (SFGs) (Glover and Law, 2001) including the anterior MTL. Compared with other imaging techniques (Glover and Lai, 1998), spiral in/out methods result in less signal dropout and greater task-related activation in MTL (Preston et al., 2004), allowing targeting of structures that have proven difficult to image because of SFGs (e.g., perirhinal cortex, entorhinal cortex, and, to a lesser extent, anterior hippocampus). Recent fMRI data using this spiral in/out sequence confirm that face stimuli elicit significant BOLD responses in perirhinal cortex, entorhinal cortex, and anterior hippocampus (Preston et al., 2009).

To obtain a field map for correction of magnetic field heterogeneity, the first time frame of the functional time series was collected with an echo time 2 ms longer than all subsequent frames. For each slice, the map was calculated from the phase of the first two time frames and applied as a first-order correction during reconstruction of the functional images. In this way, blurring and geometric distortion were minimized on a per-slice basis. In addition, correction for off-resonance attributable to breathing was applied on a per-time frame basis using phase navigation (Pfeuffer et al., 2002). This initial volume was then discarded as well as the following two volumes of each scan (a total of $12 \mathrm{~s}$ ) to allow for T1 stabilization.

Imaging analyses. Data were preprocessed using SPM2 (Wellcome Department of Imaging Neuroscience, London, UK) and custom MATLAB routines. Functional images were corrected to account for the differences in slice acquisition times by interpolating the voxel time series using sinc interpolation and resampling the time series using the center slice as a reference point. Functional volumes were then realigned to the first volume in the time series to correct for motion. A mean $\mathrm{T} 2{ }^{*}$-weighted volume was computed during realignment, and the T2-weighted anatomical volume was coregistered to this mean functional volume. As next described, the functional data were analyzed at the group level in two ways, a region of interest (ROI)-level analysis in native space and a voxellevel analysis in group-normalized space.

The first set of group analyses was performed using anatomically defined ROIs for the MTL and functionally defined ROIs for face-sensitive voxels in fusiform gyrus. For each participant, anatomically defined ROIs were demarcated on the T2-weighted, high-resolution in-plane structural images, using techniques adapted for analysis and visualization of MTL subregions (Amaral, 1990; Insausti et al., 1998; Pruessner et al., 2000, 2002; Zeineh et al., 2000, 2003; Preston et al., 2009). The 22 3-mmthick slices covered the entire MTL in all participants; eight MTL subregions were defined in each hemisphere and spanned, on average, 20 of the 22 slices. The hippocampal subfields (dentate gyrus/ $\mathrm{CA}_{2 / 3}, \mathrm{CA}_{1}$, and subiculum) within the body of the hippocampus typically spanned 12 slices. Because the hippocampal subfields cannot be delineated in the most anterior and posterior extents of the hippocampus at the resolution used, anterior hippocampal and posterior hippocampal ROIs (inclusive of all subfields) were also demarcated on the most rostral and caudal one to two slices of the hippocampus, respectively (Zeineh et al., 2003; Preston et al., 2009). These regions approximately correspond to Montreal Neurological Institute coordinates of $y=0$ to $y=-6$ for the anterior hippocampus and $y=-33$ to $y=-40$ for the posterior hippocampus (Preston et al., 2009). In addition to the five hippocampal ROIs, three MTL cortical ROIs were anatomically defined-perirhinal (PRc), parahippocampal (PHc), and entorhinal cortex (ERc). Finally, to assess activation in face-sensitive voxels in fusiform gyrus, functional ROIs in the left and right fusiform were created for each participant, defined as voxels that were active ( $p<0.05$, five-voxel extent threshold) during the viewing of faces on $30 \mathrm{~s}$ delay trials [i.e., the contrast of study and probe periods $>$ baseline; see general linear model (GLM) details below]. These functional ROIs were defined using both correct and incorrect $30 \mathrm{~s}$ trials and thus were not biased to reveal differences between these conditions.

For the ROI analyses, a finite impulse response model was applied to the unsmoothed individual subject data, wherein MarsBaR (Brett et al., 2002) was used to extract the percent signal change estimates from the 30 s delay trials for TRs $1-11$ ( $0-44$ s posttrial onset) for each condition of interest, averaged across all voxels in each anatomically defined MTL region and in each functionally defined fusiform region. Group-level repeated-measures ANOVA was used to test for differences in BOLD activity between correct and incorrect trials in each of the ROIs. Where appropriate, $\alpha$-level adjustment was computed using a Huynh-Feldt correction for nonsphericity. Because two subjects performed extremely well, with $<10$ incorrect trials on the 30 s DMS task, data from these subjects were not submitted to the analyses that included accuracy (correct vs incorrect) as a factor. Percent signal change during the DMS task was computed for the study phase (TR 2 , corresponding to $4-8$ s posttrial onset), delay phase (mean across TRs $4-8$, corresponding to 12-32 $\mathrm{s}$ posttrial onset), and probe phase (TR 10, corresponding to $36-40 \mathrm{~s}$ posttrial onset). In addition to accuracy, hemisphere (left vs right) was included as a within-subjects factor in all analyses but did not interact significantly with any effect of interest (values of $p>0.3$ ) and thus is not considered in Results. Because performance on the low-confidence trials was near chance, analysis of memory accuracy was restricted to correct trials on which participants were "confident" (i.e., the accuracy contrast compared high-confidence correct trials vs all incorrect trials).

To implement voxel-level group analyses, a second set of analyses was performed on normalized and modestly smoothed $(2 \mathrm{~mm}$ full-width at half-maximum filter) data. Specifically, each participant's anatomically defined MTL ROIs were aligned with those of a representative "target" subject using a diffeomorphic deformation algorithm implemented in the freeware package MedINRIA (version 1.7.0; Asclepios Research Team, Sophia Antipolis, France), which uses a biologically plausible, diffeomorphic transformation that respects the boundaries dictated by the ROIs. Accordingly, all participants' MTL subregions were warped into a common space, in a manner that maintains the between-region boundaries. The transformation matrix generated from the anatomical data was then applied to the first-level statistical contrast maps, which enabled second-level (group) statistical analyses. Compared with traditional whole-brain normalization techniques, this ROI alignment or "ROI-AL-Demons" approach results in more accurate correspondence of MTL subregions across subjects and higher statistical sensitivity (Stark and Okado, 2003; Kirwan et al., 2007; Yassa and Stark, 2009).

For the voxel-level analyses, data were first modeled at the individual participant level according to the GLM and accounting for the intrinsic autocorrelation in fMRI data. The statistical model also included regressors of no-interest that accounted for effects of scan session, linear drift, and motion. As with the ROI-based analyses, the $4 \mathrm{~s}$ delay trials were modeled and included in the GLM, but only data from the 30 s delay trials were further analyzed, as our hypotheses about MTL delay period activity could only be assessed during the $30 \mathrm{~s}$ trials. In the main GLM analysis, the $30 \mathrm{~s}$ delay trials were modeled using separate event regressors for study and probe periods, and a $30 \mathrm{~s}$ epoch for the delay period. Trials were modeled according to the participants' performance, resulting in separate regressors for confident correct (hereafter termed "highconfidence"), unconfident correct (hereafter, "low confidence"), and in- 

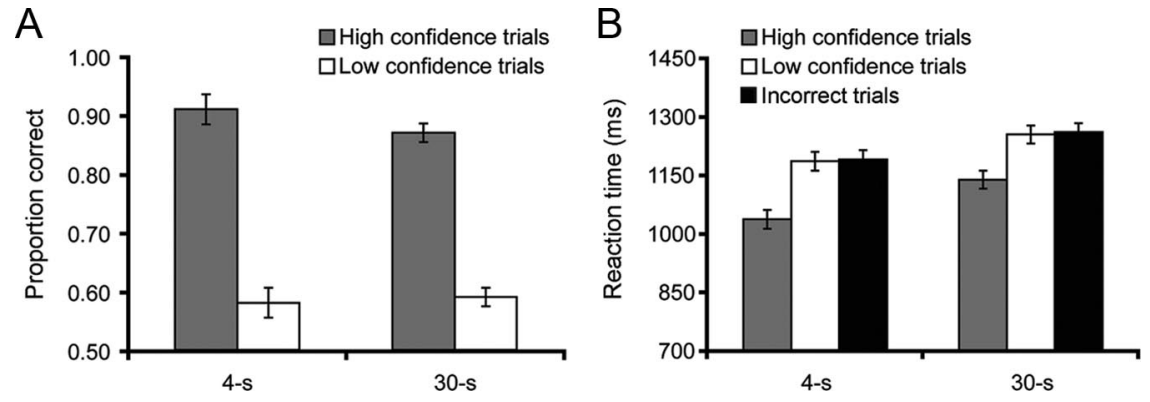

Figure 2. Behavioral performance on the DMS task. $A$, DMS accuracy is plotted by delay duration and recognition confidence. $\boldsymbol{B}$, Reaction times are plotted by delay duration for high-confidence correct, low-confidence correct, and incorrect trials. In all figures, error bars reflect within-subject SE.

correct trials for the study, delay, and probe periods. A second GLM was specified to examine linear trends during the delay period. This model was identical with the first, except that an additional regressor of interest was added that tested for linear increases (or decreases) across the $30 \mathrm{~s}$ delay period (TRs $4-8$, corresponding to $12-32 \mathrm{~s}$ posttrial onset). For both models, group-level statistical maps were created using an uncorrected voxelwise threshold of $p<0.001$, and to correct for multiple comparisons, a small-volume correction (SVC) over the MTL was used to establish a cluster-level corrected threshold of $p_{\text {svc }}<0.05$. The MTL mask used for the SVC was created by (1) combining the eight anatomically defined ROIs (after ROI-AL transformation) into a single volume for each participant, and then (2) combining all participants' MTL volumes into a single image. Thus, the mask was generated from the obtained anatomical data and was conservatively constructed to include every voxel that had been assigned to the MTL in at least one of the participants.

\section{Results}

\section{Behavioral performance}

A repeated-measures ANOVA on DMS task accuracy, with factors of delay duration (4 vs $30 \mathrm{~s}$ ) and response confidence (high vs low), revealed (1) no effect of delay duration $(p=0.55)$, (2) superior accuracy on high- versus low-confidence trials $\left(F_{(1,19)}=161.86 ; p<0.001\right)($ Fig. $2 A)$, and (3) no delay by confidence interaction $\left(F_{(1,19)}=2.21 ; p=0.15\right)$. Reaction time analyses on correct trials revealed an effect of delay duration $\left(F_{(1,19)}=15.26\right.$; $p=0.001)$, an effect of confidence $\left(F_{(1,19)}=26.86 ; p<0.001\right)$, but no interaction $\left(F_{(1,19)}=1.19 ; p=0.29\right)$ (Fig. $\left.2 B\right)$. Furthermore, a comparison of high-confidence correct trials with incorrect trials revealed faster reaction times during the former $\left(t_{(17)}=-3.47 ; p=\right.$ 0.003 ), indicating that any observation of greater $\mathrm{AMRI}$ activation during high-confidence correct versus incorrect trials is not attributable to longer time on task at probe during high-confidence correct trials.

\section{fMRI region of interest group analyses}

\section{Delay period MTL activity predicts DMS success}

Previous standard-resolution fMRI studies have revealed delay period MTL activation using short-delay intervals (delay periods ranged from 7 to $13 \mathrm{~s}$ ) (Ranganath and D'Esposito, 2001; Schon et al., 2004; Nichols et al., 2006; Axmacher et al., 2007), although the relationship between this delay period activation in human MTL and performance after the brief retention interval is unknown. Moreover, because previous fMRI studies targeting MTL delay period responses used novel, trial-unique stimuli (cf. Piekema et al., 2006), it is unclear whether human MTL subregions demonstrate delay period activation when using repeated (non-trial-unique) stimuli. In the nonhuman, recent electrophysiological data from rodent hippocampus have revealed dynamic changes in hippocampal activation over a $30 \mathrm{~s}$ delay period that relate to task accuracy (Deadwyler and Hampson, 2004). Moreover, observations of persistent activation in rodent and macaque entorhinal cortex during DMS (Suzuki et al., 1997; Young et al., 1997) suggest that entorhinal cortex is engaged during DMS delay periods, and lesion data indicate that the cortex in and surrounding the rhinal sulcus in the macaque is necessary for DMS performance with trial-unique stimuli but not with repeated stimuli (Eacott et al., 1994).

To examine the relationship between delay period activation and DMS performance using familiarized stimuli, we tested whether MTL subregions display sustained activation over a $30 \mathrm{~s}$ delay and whether the magnitude of activation during the delay period correlates with subsequent memory performance at the probe (Paller and Wagner, 2002). To the extent that visual object stimuli, such as faces, are preferentially encoded in perirhinal cortex (Buffalo et al., 2006; Preston et al., 2009) and that the sustained allocation of attention to face representations during delays serves to drive sustained responses in the MTL, then we predicted that delay period activation would be observed in perirhinal and entorhinal cortex, as well as in anterior hippocampal regions that differentially receive perirhinal inputs via entorhinal cortex (Witter et al., 1989; Witter and Amaral, 1991; Suzuki and Amaral, 1994a; Small, 2002). Moreover, to the extent that active maintenance and/or MTL binding mechanisms contribute to mnemonic performance on the DMS task, then we predicted that greater delay period activity would be associated with successful versus unsuccessful task performance.

Consistent with these predictions, three regions within the MTL exhibited significantly greater delay period activity on highconfidence correct trials than on incorrect trials (Fig. 3). Specifically, perirhinal cortex (Fig. $3 A, D)\left(F_{(1,17)}=8.45 ; p=0.01\right)$ and entorhinal cortex (Fig. $3 B, E)\left(F_{(1,17)}=6.60 ; p=0.02\right)$ both showed greater delay period activity on subsequently correct versus incorrect trials, as did the anterior hippocampus (Fig. 3C,F) $\left(F_{(1,17)}=6.89 ; p=0.018\right)$. These differences in delay period activity do not reflect a carryover of differential MTL activation during the study period, as the magnitude of study period activation in perirhinal, entorhinal, and anterior hippocampus did not significantly differ between subsequently correct versus incorrect trials ( $p=0.66, p=0.97$, and $p=0.98$, respectively). As such, the observed relationship between delay period MTL activation and DMS accuracy suggests that processes engaged during the retention interval impact later performance.

In the nonhuman, selective lesions to rhinal cortex produce impairments on DMS performance when delays exceed $5 \mathrm{~s}$ (Gaffan and Murray, 1992; Baxter and Murray, 2001), whereas deficits after selective hippocampal lesions are more variable (Alvarez et al., 1995; Murray and Mishkin, 1998). In the current study, beyond the anterior hippocampus ROI (described above), no other hippocampal subregion demonstrated a sustained response that varied with subsequent memory success. In particular, although delay period activity was significantly greater than study and probe phase activity in the dentate gyrus/ $/ \mathrm{A}_{2 / 3}$ and $\mathrm{CA}_{1}\left(F_{(1,17)}=9.06, p=0.008\right.$; and $F_{(1,17)}=5.60, p=0.03$, respectively), suggesting that these regions were engaged during the delay period, the magnitude of this delay period response did not significantly differ during correct versus incorrect trials (values of $p>0.55)$. 
Time-dependent increase in delay period activity

Previous fMRI studies have identified sustained delay period activity in the ventral visual stream, including the fusiform gyrus, during short-delay tasks with face stimuli (Courtney et al., 1997; Haxby et al., 2000; Jiang et al., 2000). To examine whether BOLD activity in the MTL and fusiform cortex displays a dynamic change over the delay period, we explored whether activation in any of the anatomically defined MTL ROIs or the functionally defined fusiform ROIs either linearly increased or decreased over the course of the retention interval. Our motivation for investigating increasing and decreasing patterns of activity was twofold. First, in previous studies that used short-delay recognition tasks (7-13 s delay periods), it was often the case that the delay period activity was highest at the beginning of the delay period, raising the possibility that the activity may be driven at least partially by the encoding event (Ranganath and D’Esposito, 2001; Ranganath et al., 2005; Nichols et al., 2006). Second, previous electrophysiological data have revealed dynamic changes in single-unit firing rates over the course of extended delay periods both in the MTL and prefrontal cortex (Quintana and Fuster, 1999; Freedman et al., 2001; Deadwyler and Hampson, 2004). For example, Quintana and Fuster (1999) observed that some prefrontal neurons behave like conventional "workingmemory cells," whereas others demonstrate an increase in response over the course of delay, compatible with an anticipatory or "preparatory set" effect (Fuster, 2001).

In the present study, both parahippocampal cortex and fusiform gyrus demonstrated a significant increase in BOLD activity across the $30 \mathrm{~s}$ delay period (Fig. 4). Specifically, although neither region demonstrated differential delay period activity on correct versus incorrect trials ( $p=0.25$ and $p=0.97$, respectively), there was a significant effect of time (TRs $4-8$ corresponding to $12-32$ s posttrial onset) in parahippocampal cortex $\left(F_{(4,68)}=12.97 ; p<\right.$ $0.001)$ and fusiform gyrus $\left(F_{(4,68)}=9.32 ; p<0.001\right)$. Lineartrend analyses revealed a linear increase in the magnitude of activation in parahippocampal cortex $\left(F_{(1,17)}=23.38 ; p<0.001\right)$ and fusiform gyrus $\left(F_{(1,17)}=23.06 ; p<0.001\right)$ as the retention interval progressed, such that activation reached a delay period maximum just before onset of the probe.

The linear increase in parahippocampal cortex in the absence of a success effect appeared to qualitatively differ from the absence of increases in perirhinal cortex and entorhinal cortex in the presence of success effects. Confirming this possibility, ANOVA comparing the pattern of delay period activation in parahippocampal cortex to that in (1) perirhinal cortex and (2) entorhinal cortex revealed significant region [(1) PHc/PRc; (2) PHc/ERc] by time interactions $\left[(1) F_{(4,68)}=4.39, p=0.003 ;(2)\right.$ $\left.F_{(4,68)}=4.35, p=0.009\right]$, suggesting a functional dissociation between parahippocampal cortex and both perirhinal and entorhinal cortex. In contrast, the pattern of delay period activation did
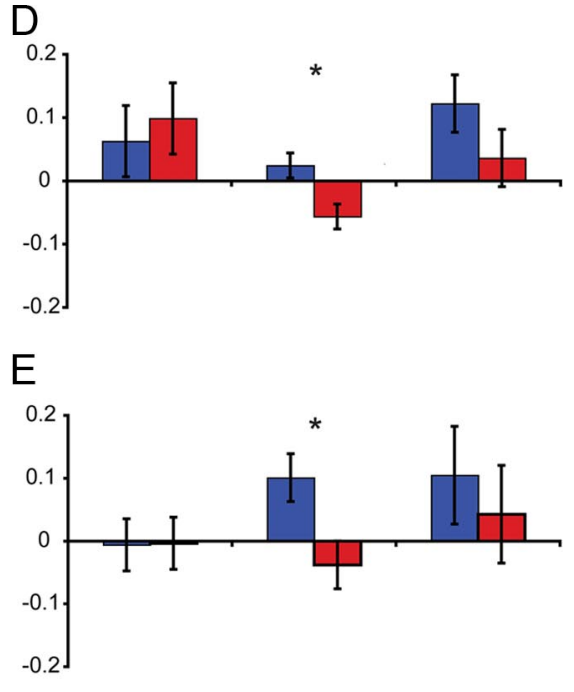

$\mathrm{F}$

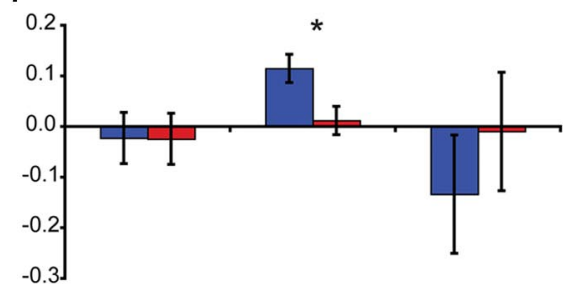

Study Delay Probe Time (s)

nal cortex, and anterior hippocampus is correlated with subsequent memory performance at probe. The left panels $(\boldsymbol{A}-\boldsymbol{C})$ display the average percent signal change across the entire trial for data from each anatomically defined region of interest. The right panels $(\boldsymbol{D}-\boldsymbol{F})$ depict the percent signal change during the three task periods [study (TR 2, corresponding to 4-8s posttrial onset), delay (mean across TRs $4-8$, corresponding to 12-32 s posttrial onset), and probe (TR 10, corresponding to $36-40$ s posttrial onset)]. * $p<0.05$.

not differ between perirhinal and entorhinal cortex (region by time, $p=0.97)$.

\section{Performance-related activation during the probe}

The preceding analyses indicate that delay period activation in perirhinal cortex, entorhinal cortex, and anterior hippocampus predicts later probe decision success, whereas positively ramping delay period activation in parahippocampal cortex and fusiform gyrus suggests engagement of anticipatory processes. We next sought to explore whether MTL activation during the probe presentation differed across correct versus incorrect decisions. It has been recently hypothesized that MTL responses to probe stimuli are greater when the probe matches the contents of internally generated goal states (Hannula and Ranganath, 2008; Duncan et al., 2009). Accordingly, we expected to find a greater BOLD response during the probe phase on trials in which the participant correctly identified the studied face than when the participant was unable to identify the studied face.

Consistent with this prediction, probe period activity was significantly greater for correct versus incorrect trials in parahippocampal cortex (Fig. $4 A, C)\left(F_{(1,17)}=6.79 ; p=0.018\right)$, with a significant accuracy (correct/incorrect) by phase (study, delay, probe) interaction $\left(F_{(2,34)}=8.36 ; p=0.001\right)$ indicating that the accuracy effect was specific to the probe period. The $\mathrm{CA}_{1}$ subregion of the hippocampus also demonstrated a probe period success effect $\left(F_{(1,17)}=4.46 ; p=0.05\right)$, and similar trends were observed in dentate gyrus $/ \mathrm{CA}_{2 / 3}\left(F_{(1,17)}=3.66 ; p=0.073\right)$ and subiculum $\left(F_{(1,17)}=3.14 ; p=0.094\right)$. Moreover, $\mathrm{CA}_{1}$ and den- 

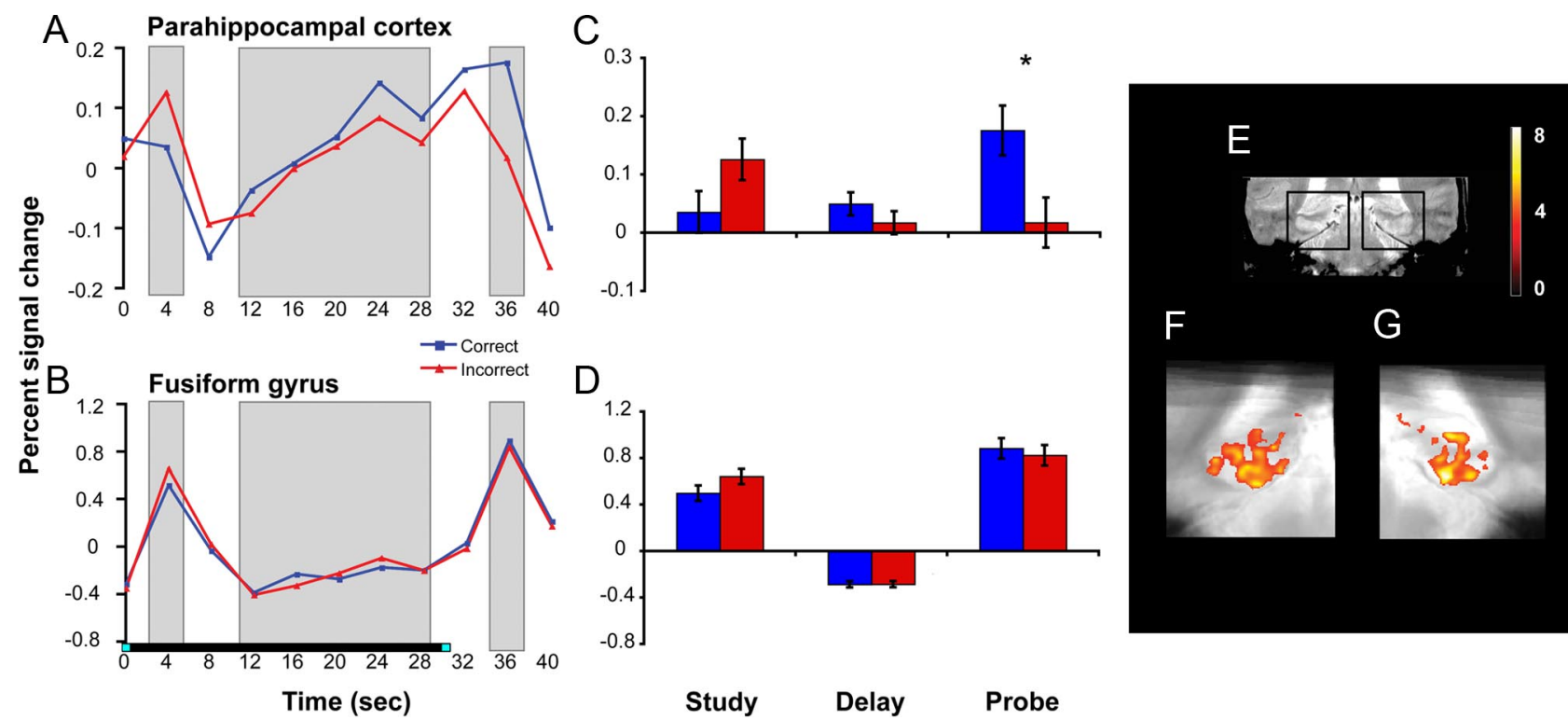

Study Delay Probe

Figure 4. Linearly increasing delay period activity and performance-related probe period activity in parahippocampal cortex and fusiform gyrus. The left $(\boldsymbol{A}, \boldsymbol{B})$ and middle $(\boldsymbol{C}, \boldsymbol{D})$ panels display the BOLD response in anatomically defined parahippocampal cortex and functionally defined fusiform gyrus. The delay period response linearly increased with time $(\boldsymbol{A}, \boldsymbol{B})$, and the probe period response was greater on correct versus incorrect trials $\left({ }^{*} p<0.05\right)$. $\boldsymbol{E}$ displays a coronal slice through a representative participant's T2-weighted in-plane anatomical image, with black boxes demarcating the MTL regions featured in $\boldsymbol{F}$ and $\boldsymbol{G} . \boldsymbol{F}$ and $\boldsymbol{G}$ display group-level voxel-based analyses revealing clusters in left and right parahippocampal cortex and posterior hippocampus that demonstrated linearly increasing activity across the delay period (voxel threshold: $p<0.001$, uncorrected; cluster threshold: $p<0.05$, corrected). The underlay is the group mean-transformed in-plane structural image.

tate gyrus/ $\mathrm{CA}_{2 / 3}$ showed significant accuracy (correct/incorrect) by phase (study, delay, probe) interactions $\left(F_{(2,34)}=4.63, p=\right.$ 0.028 ; and $F_{(2,34)}=4.23, p=0.035$, respectively).

Performance-independent across-region functional dissociations The preceding analyses indicate that the delay period response in perhinal and entorhinal cortex dissociates from that in parahippocampal cortex, with the former regions demonstrating sustained delay period activation that differed according to subsequent recognition performance and the latter region demonstrating an anticipatory delay period response that was insensitive to performance. Although both perirhinal and entorhinal cortex demonstrated similar performance-related delay period activation (region by accuracy interaction on delay period activity, $p=0.19$ ), additional analysis suggested a performanceindependent functional differentiation between perirhinal and entorhinal cortex. Specifically, after collapsing across correct and incorrect trials, ANOVA revealed a region (PRc/ERc) by phase (study, delay, probe) interaction $\left(F_{(2,34)}=5.49 ; p=0.009\right)$ : perirhinal cortex was more active than entorhinal cortex during study $\left(F_{(1,17)}=6.68 ; p=0.019\right)$, whereas entorhinal cortex was more active than perirhinal cortex during delay $\left(F_{(1,17)}=5.14\right.$; $p=0.037)$. This crossover interaction reveals a differentially greater transient response in perirhinal cortex during face encoding, perhaps related to stimulus-level coding (Preston et al., 2009), and a greater persistent response in entorhinal cortex during the delay period, consistent with recent hypotheses regarding the ability of entorhinal cortex to maintain representations across delays (Hasselmo and Stern, 2006).

Within the hippocampal subfields, although there was a similar pattern of performance-related delay period activation across the three regions (all region by accuracy interactions on delay period activity: values of $p>0.46$ ), again performanceindependent interactions were observed. Specifically, region by phase interactions dissociated dentate gyrus/ $\mathrm{CA}_{2 / 3}$ from both $\mathrm{CA}_{1}\left(F_{(2,34)}=21.72 ; p<0.001\right)$ and subiculum $\left(F_{(2,34)}=29.45\right.$; $p<0.001)$. The former dissociation was attributable to dentate gyrus/ $\mathrm{CA}_{2 / 3}$ demonstrating higher activity during the study and delay phases $\left(F_{(1,17)}=12.64, p=0.002\right.$; and $F_{(1,17)}=18.26, p=0.001$, respectively), whereas $\mathrm{CA}_{1}$ demonstrated higher activity at probe $\left(F_{(1,17)}=15.04 ; p=0.001\right)$. Similarly, the latter dissociation was attributable to dentate gyrus/ $\mathrm{CA}_{2 / 3}$ demonstrating higher activity during the early portion of the delay period $\left(F_{(1,17)}=5.15 ; p=\right.$ $0.037)$, whereas subiculum demonstrated higher activity at probe $\left(F_{(1,17)}=33.00 ; p>0.001\right)$. Although not performance related, these dissociations suggest a differential role of dentate gyrus/ $\mathrm{CA}_{2 / 3}$ in earlier periods of DMS task execution, relative to $\mathrm{CA}_{1}$ and subiculum, paralleling previous high-resolution fMRI studies of human hippocampal subfield function during declarative memory encoding and retrieval (Zeineh et al., 2003; Eldridge et al., 2005).

\section{fMRI voxel-level group analyses}

While providing increased anatomical precision, the preceding anatomically defined ROI analyses may be insensitive to more focal effects within the MTL, because for each ROI the analysis undoubtedly pools across responsive and nonresponsive voxels. In addition, because the data are pooled across all voxels in an ROI, possible topographical information within a region is lost. Accordingly, to further characterize the response of MTL subregions during the study, delay, and probe phases of the DMS task, we used the "ROI-AL-Demons" approach for across-subject normalization of the obtained MTL data, as this approach results in more accurate correspondence of MTL subregions across subjects and higher voxel-level statistical sensitivity (Stark and Okado, 2003; Kirwan et al., 2007; Yassa and Stark, 2009).

As with the preceding ROI-level analyses, voxel-level group analysis of study period activity did not reveal significant clusters of MTL voxels when contrasting subsequently correct versus incorrect trials. The absence of a significant difference between study phase activity according to later memory performance stands in contrast with an extensive literature documenting that 
MTL encoding activity differs according to subsequent memory performance at long delays (Brewer et al., 1998; Wagner et al., 1998; Henson et al., 1999; Davachi et al., 2003; Ranganath et al., 2004) (for review, see Davachi, 2006). We note that the present design differs from previous studies in at least one potentially relevant way. Specifically, the stimuli here were prefamiliarized and not trial unique, whereas previous subsequent memory studies typically used novel, trial-unique stimuli. The use of familiarized, repeating stimuli is known to decrease MTL BOLD activity (Stern et al., 1996; Kirchhoff et al., 2000; O'Kane et al., 2005), which may diminish the impact of stimulus encoding processes on later performance, shifting the relevant variance to processes engaged during the $30 \mathrm{~s}$ delay period. Moreover, the use of an immediate recognition memory test may have also differentially emphasized a relationship between memory behavior and delay period neural processes.

Indeed, as with the ROI-level analyses, voxel-level analysis revealed subsequent memory effects in multiple MTL regions during the delay period (Fig. 5). Specifically, in the left MTL, we observed a cluster in entorhinal cortex (Fig. $5 B)(k=101$; cluster $_{\text {volume }}=48.5 \mathrm{~mm}^{3} ; p<0.001$, uncorrected; $\left.p_{\mathrm{svc}}=0.037\right)$. In the right MTL, we observed a cluster in the anterior portion of the hippocampus (Fig. $5 C)(k=$ 119 ; cluster $_{\text {volume }}=57.1 \mathrm{~mm}^{3} ; p<0.001$, uncorrected; $p_{\text {svc }}=0.018$ ), and a separate cluster in entorhinal cortex, falling near perirhinal cortex (Fig. $5 D)(k=43$; cluster $_{\text {volume }}=20.6 \mathrm{~mm}^{3} ; p<0.001$, uncorrected; $\left.p_{\mathrm{svc}}=0.237\right)$. Furthermore, clusters in left parahippocampal cortex and left posterior hippocampus (Fig. $4 F$ ) $\left(k=3026\right.$; cluster volume $=1453.5 \mathrm{~mm}^{3}$; $p<0.001$, uncorrected; $p_{\mathrm{svc}}<0.001$; and $k=362$; cluster $_{\text {volume }}=157.44 \mathrm{~mm}^{3} ; p<$ 0.001 , uncorrected; $p_{\mathrm{svc}}<0.001$, respectively) and in right parahippocampal cortex extending into right posterior hippocampus (Fig. 4G) $(k=3125$; cluster $_{\text {volume }}=1500 \mathrm{~mm}^{3} ; p<0.001$, uncorrected; $\left.p_{\mathrm{svc}}<0.001\right)$ showed a significant linear increase in delay period activity. Thus, these voxel-level group analyses confirmed the delay period findings observed in the anatomically defined ROI-level group analysis and further revealed a positively ramping anticipatory response in posterior hippocampus.

Finally, we tested for voxels that demonstrated greater activation on correct versus incorrect trials during the probe phase of the DMS task. Again, paralleling our anatomically defined ROI analysis, we found clusters in the mid to posterior part of the left and right hippocampus, situated predominantly in dentate gyrus/CA $\mathrm{CA}_{2 / 3}$ (Fig. $6 \mathrm{~B}$ ) $\left(k=160\right.$; cluster ${ }_{\text {volume }}=76.8 \mathrm{~mm}^{3} ; p<0.001$, uncorrected; $p_{\mathrm{svc}}=$ 0.009) (Fig. 6C) $\left(k=108\right.$; cluster ${ }_{\text {volume }}=51.8 \mathrm{~mm}^{3} ; p<0.001$, parahippocampal clusters.
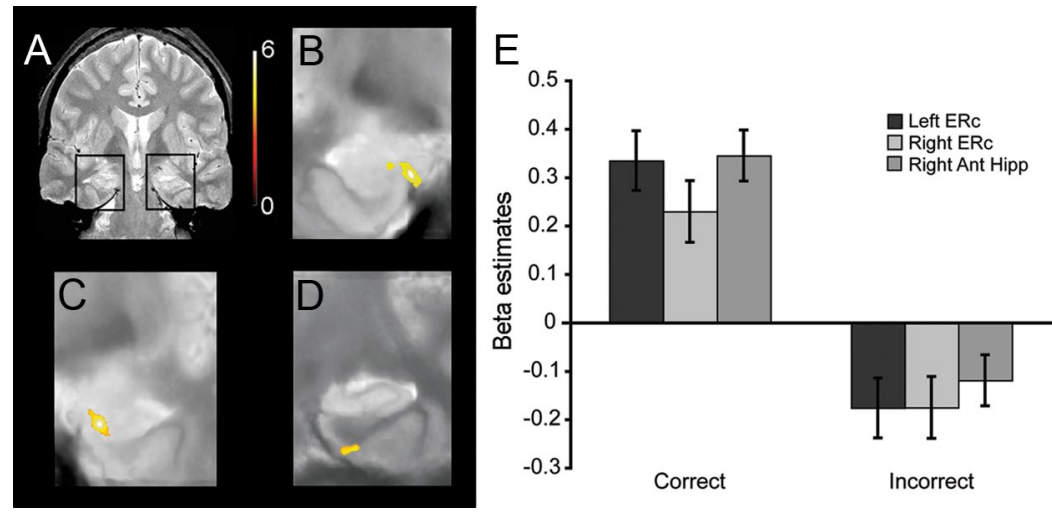

Correct

Incorrect

Figure 5. Group-level statistical maps of MTL regions demonstrating performance-related delay period activity (highconfidence correct $>$ incorrect trials). $A$, A coronal slice through a representative participant's T2-weighted in-plane anatomical image, demarcating the MTL regions featured in $\boldsymbol{B}-\boldsymbol{D}$. Significant clusters (voxel threshold, $p<0.001$, uncorrected; cluster threshold, $p<0.05$, corrected) of performance-related delay period activity in left entorhinal cortex, near the anterior extent 0 the hippocampus $(\boldsymbol{B})$, right anterior hippocampus (C), and right entorhinal cortex, near perirhinal cortex (D). $\boldsymbol{E}$, Mean $\beta$ estimates averaged across voxels within the left and right clusters.
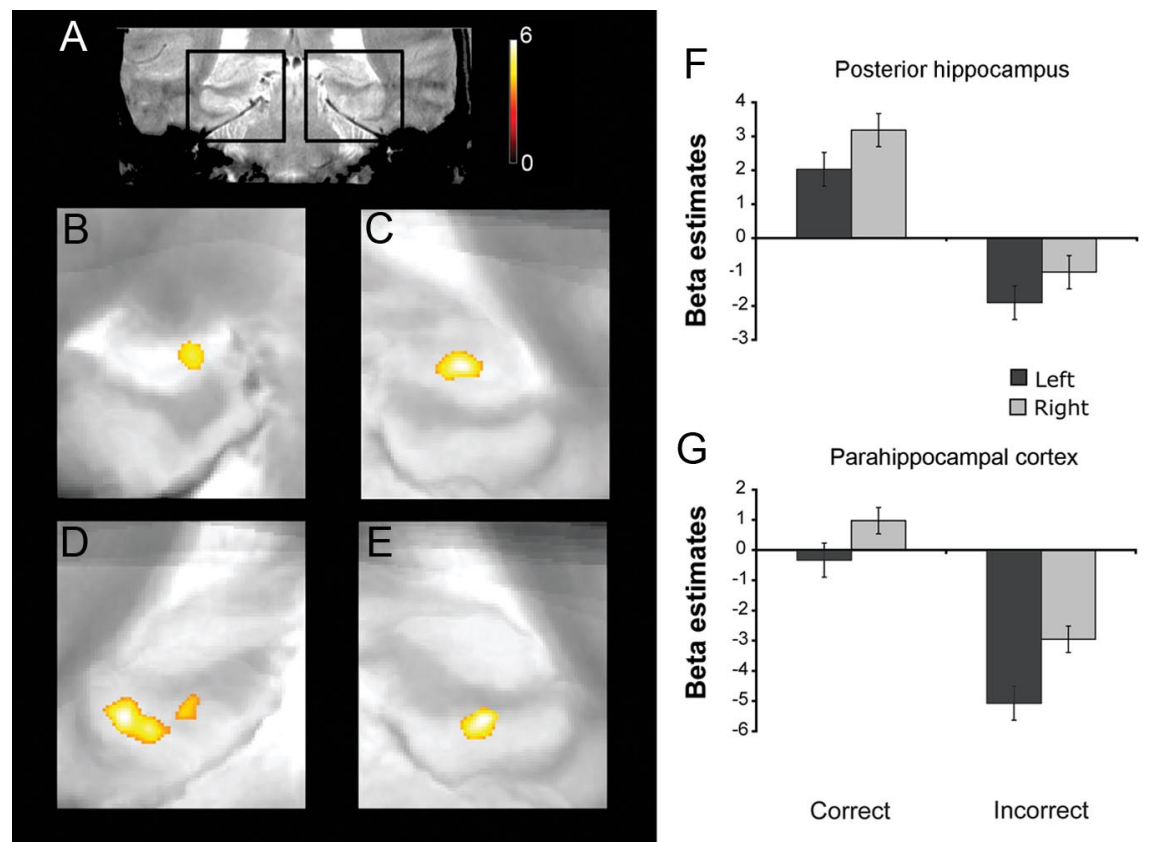

Correct Incorrect

Figure 6. Group-level statistical maps of MTL regions demonstrating performance-related probe period activity (highcorrect $>$ incorrect trials). A, A coronal slice through a representative participant's T2-weighted in-plane anatomical mage, demarcating the MTL regions featured in $\boldsymbol{B}-\boldsymbol{E}$. Significant clusters (voxel threshold, $p<0.001$, uncorrected; cluster hreshold, $p<0.05$, corrected) of performance-related probe period activity in left and right mid to posterior hippocampus ( $B, C$ and posterior parahippocampal cortex $(\boldsymbol{D}, \boldsymbol{E}) . \boldsymbol{F}, \boldsymbol{G}$, Mean $\beta$ estimates averaged across voxels within the posterior hippocampal and

uncorrected; $p_{\mathrm{svc}}=0.027$, respectively), as well as two clusters in left parahippocampal cortex (Fig. $6 D)\left(k=377\right.$; luster $_{\text {volume }}=181.0$; $p<0.001$ uncorrected; $p_{\text {svc }}<0.001$; and $k=159$; cluster $_{\text {volume }}=$ $76.3 \mathrm{~mm}^{3} ; p<0.001$, uncorrected; $\left.p_{\mathrm{svc}}=0.009\right)$ and one in right parahippocampal cortex (Fig. $6 E)\left(k=157\right.$; cluster $_{\text {volume }}=75.4$ $\mathrm{mm}^{3} ; p<0.001$, uncorrected; $\left.p_{\mathrm{svc}}=0.007\right)$.

\section{Discussion}

The present study revealed two patterns of delay period activity-sustained and anticipatory-across distinct MTL subregions during DMS task performance, suggesting that multiple MTL mechanisms contribute to short-delay recognition memory. First, during the delay, perirhinal cortex, entorhinal 
cortex, and anterior hippocampus demonstrated greater sustained activity during subsequently correct versus incorrect trials. Because the stimuli were familiarized, non-trial-unique items, this finding indicates that delay period responses in human MTL are both performance related and not restricted to conditions favoring novelty encoding. Moreover, use of a $30 \mathrm{~s}$ unfilled retention interval ensures that the observed BOLD response was unambiguously attributable to delay period processes. Second, activation in parahippocampal cortex, posterior hippocampus, and fusiform gyrus demonstrated linear increases over the $30 \mathrm{~s}$ delay, suggesting an anticipatory response predictive of the probe. Consistent with this interpretation, parahippocampal cortex and hippocampal subfields demonstrated greater probe period activation on correct versus incorrect trials. The theoretical implications of these findings are discussed in turn.

\section{Sustained delay period activity predicts DMS performance}

The observation that delay period BOLD activity in human MTL is predictive of immediate-recognition performance extends previous electrophysiological, lesion, and fMRI data that suggest a role for the MTL in successful short-delay DMS task performance. First, this finding complements intracranial recording data from humans (Axmacher et al., 2007) and nonhumans (Lehky and Tanaka, 2007) that revealed activity in perirhinal cortex when subjects perform tasks that require memory of a visual stimulus after a short delay, and lesion and recording studies in rodents (Otto and Eichenbaum, 1992; Young et al., 1997; Van Cauter et al., 2008) and monkeys (Baylis and Rolls, 1987; Meunier et al., 1993, 1996; Suzuki et al., 1993) that suggest that entorhinal, perirhinal, and parahippocampal cortical areas are involved in delayed-match and delayed-non-match-to-sample performance. Second, although previous fMRI studies reported delay period MTL activity that correlates with subsequent recognition performance after a long retention interval (Schon et al., 2004; Ranganath et al., 2005; Nichols et al., 2006), the present data are the first, to our knowledge, to demonstrate a relationship between trial-by-trial variability in the magnitude of sustained delay period MTL activity and immediate DMS performance [for an across-subject correlation between MTL activity and DMS task performance, see Hannula and Ranganath (2008)]. Although correlational, these data provide the strongest evidence to date that performance-related persistent activity is present in human perirhinal cortex, entorhinal cortex, and anterior hippocampus during short-delay recognition tasks.

The present improved-resolution fMRI methods also afford greater confidence in the localization of delay period responses to particular MTL subregions, including to entorhinal and perirhinal cortex. Motivated by nonhuman lesion (Otto and Eichenbaum, 1992; Eacott et al., 1994; Turchi et al., 2005) and standard-resolution human neuroimaging data (Ranganath and D'Esposito, 2001; Schon et al., 2004), Hasselmo and Stern (2006) posit a role for entorhinal (and perhaps perirhinal) cortex in mediating working memory for novel stimuli. Our data can be viewed as consistent with this proposal, as they reveal (1) greater study period activity in perirhinal versus entorhinal cortex, but greater delay period activity in entorhinal versus perirhinal cortex, and (2) delay period responses, uncontaminated by encoding-phase activity (for a discussion of this issue, see Postle, 2006), in entorhinal, perirhinal, and anterior hippocampus that correlate with immediate DMS performance, suggesting a relationship between these MTL responses and performance at short delays. At the same time, our data indicate that MTL delay period activity is not restricted to tasks using novel stimuli, as the present effects were observed using familiarized, non-trial-unique faces. Because it remains possible that performance-related delay period MTL activity might be further heightened for novel stimuli, a systematic investigation of the influence of stimulus novelty/familiarity on delay period responses would further advance understanding of how the MTL mediates recognition at short delays (Zarahn et al., 2005).

\section{Stimulus class effects in MTL cortex}

In the nonhuman, perirhinal and parahippocampal cortex receive divergent visual inputs, with ventral visual cortex projecting predominantly to perirhinal cortex and dorsal visual cortex providing major inputs to parahippocampal cortex (Suzuki and Amaral, 1994b; Burwell and Amaral, 1998; Suzuki, 2009). This differential connectivity is hypothesized to produce mnemonic distinctions between these MTL cortical regions according to stimulus class (Davachi, 2006; Eichenbaum et al., 2007; Preston and Wagner, 2007). Indeed, damage to human perirhinal cortex can result in object recognition memory deficits (Buffalo et al., 1998) and impaired visual discrimination of complex objects and faces (Barense et al., 2007; Bird et al., 2007; Taylor et al., 2007) (but see Shrager et al., 2006), whereas damage to human parahippocampal cortex can result in memory deficits for topographical and spatial stimuli (Bohbot et al., 2000; Epstein et al., 2001). Extant fMRI studies indicate that parahippocampal cortex shows a clear preference for location/scene encoding, whereas perirhinal and entorhinal cortex are active during both object and location/scene encoding (Buffalo et al., 2006; Preston et al., 2009). As such, a content-based functional gradient appears present along the rostrocaudal axis of human collateral sulcus (Litman et al., 2009).

The present dissociation between sustained delay period activity in perirhinal cortex and ramping anticipatory activity in parahippocampal cortex may partially reflect the differential coding of object (face) and spatial information during the DMS retention interval. Specifically, because the DMS task demands memory for two faces, independent of their spatial locations, one approach to task performance is to maintain representations of the presented faces across the 30 s delay. Such maintenance might occur beyond the MTL (e.g., via prefrontal-fusiform interactions) (Gazzaley et al., 2004; Postle, 2006), which would have a secondary consequence of temporally extending the inputs or drive to perirhinal cortex over the delay. From this perspective, the delay period activity in perirhinal, entorhinal, and anterior hippocampus is a marker of the maintenance of face representations in lateral cortical structures that project to anterior hippocampus through perirhinal and entorhinal cortex. This interpretation is challenged, however, by the absence of a performance-related delay period response in fusiform cortex (but see Harrison and Tong, 2009; Serences et al., 2009), which would be expected to represent the studied faces across the delay. Alternatively, the sustained delay period response in perirhinal, entorhinal, and anterior hippocampal regions may reflect representational persistence within the MTL proper, independent of drive from lateral perceptual regions. This response may reflect an extended engagement of MTL encoding/binding mechanisms that serves to associate the studied faces to the temporal context of the trial, thus affording discrimination between the target and foil during the probe.

Although parahippocampal cortex has recently been proposed to mediate domain-general context representations (Diana et al., 2007), other accounts of parahippocampal function (Epstein and Kanwisher, 1998) might suggest that the ramping 
delay period response in this region reflects spatial anticipatory responses. As the $30 \mathrm{~s}$ delay unfolds, participants likely anticipate the subsequent onset of the probe, which consisted of two faces in predictable spatial locations. In anticipation, subjects may engage top-down processes that result in a prospective attention-based gain modulation of the cortical regions coding for the upcoming class of stimuli (i.e., fusiform gyrus for faces) and their upcoming locations (i.e., parahippocampal cortex). Interestingly, parahippocampal cortex demonstrated an anticipatory delay period response and a performance-related difference in probe phase BOLD activity, suggesting that anticipatory responses impact performance at the probe (perhaps by amplifying mnemonic signal differences between the probes) (for related discussion, see Dobbins and Wagner, 2005). Future studies that relate trial-bytrial anticipatory responses to subsequent probe period responses are required to fully examine this possibility.

\section{Does the MTL mediate working memory?}

By including $4 \mathrm{~s}$ delay trials intermixed with the critical $30 \mathrm{~s}$ delay trials, and by using repeating stimuli, the present study was designed to encourage active maintenance, a process essential to working memory. We stress, however, that the present performance-related delay period activity within the MTL during this short-delay task does not demand the conclusion that MTL mechanisms support working memory maintenance processes (Shrager et al., 2008). Rather, this activity could reflect the sustained engagement of MTL-dependent long-term memory processes that foster face-context encoding that subsequently supports performance (Jonides et al., 2008). Alternatively, the sustained MTL activity could be a consequence of temporally extended inputs to the MTL attributable to the maintenance of representations by lateral temporal and frontal cortical regions. Interestingly, a recent study using face stimuli found that prefrontal-fusiform connectivity during the delay period decreased as a function of load, whereas MTL-fusiform connectivity increased (Rissman et al., 2008), suggesting that these structures play complementary roles depending on task demands. Future studies that attempt to relate MTL delay period responses to lateral cortical correlates of working memory maintenance may serve to further disambiguate the role of MTL structures during performance of short-delay tasks.

\section{Summary}

The current study of short-delay recognition memory provides evidence that sustained and anticipatory functional responses are observed in distinct MTL subregions. Performance-related delay period MTL activity may reflect sustained maintenance of stimulus-specific representations or may serve to bind stimuli to context, whereas ramping delay period activity may reflect anticipatory responses that facilitate probe phase discrimination. Although it was once believed that the MTL solely performs functions in the service of long-delay memory, the present data add to an emerging literature implicating MTL processes in recognition performance even at short delays.

\section{References}

Alvarez P, Zola-Morgan S, Squire LR (1995) Damage limited to the hippocampal region produces long-lasting memory impairment in monkeys. J Neurosci 15:3796-3807.

Amaral D (1990) The human hippocampal formation. In: The human nervous system (Paxinos G, Mai JK, eds), pp 711-755. San Diego: Academic.

Axmacher N, Mormann F, Fernández G, Cohen MX, Elger CE, Fell J (2007) Sustained neural activity patterns during working memory in the human medial temporal lobe. J Neurosci 27:7807-7816.
Barense MD, Gaffan D, Graham KS (2007) The human medial temporal lobe processes online representations of complex objects. Neuropsychologia 45:2963-2974.

Baxter MG, Murray EA (2001) Opposite relationship of hippocampal and rhinal cortex damage to delayed nonmatching-to-sample deficits in monkeys. Hippocampus 11:61-71.

Baylis GC, Rolls ET (1987) Responses of neurons in the inferior temporal cortex in short term and serial recognition memory tasks. Exp Brain Res 65:614-622.

Bird CM, Shallice T, Cipolotti L (2007) Fractionation of memory in medial temporal lobe amnesia. Neuropsychologia 45:1160-1171.

Bohbot VD, Allen JJ, Nadel L (2000) Memory deficits characterized by patterns of lesions to the hippocampus and parahippocampal cortex. Ann N Y Acad Sci 911:355-368.

Brainard DH (1997) The Psychophysics Toolbox. Spat Vis 10:433-436.

Brett M, Anton JL, Valabregue R, Poline JB (2002) Region of interest analysis using an SPM toolbox. Paper presented at Eighth International Conference on Functional Mapping of the Human Brain, Sendai, Japan, June.

Brewer JB, Zhao Z, Desmond JE, Glover GH, Gabrieli JD (1998) Making memories: brain activity that predicts how well visual experience will be remembered. Science 281:1185-1187.

Buffalo EA, Reber PJ, Squire LR (1998) The human perirhinal cortex and recognition memory. Hippocampus 8:330-339.

Buffalo EA, Bellgowan PS, Martin A (2006) Distinct roles for medial temporal lobe structures in memory for objects and their locations. Learn Mem 13:638-643.

Burwell RD, Amaral DG (1998) Cortical afferents of the perirhinal, postrhinal, and entorhinal cortices of the rat. J Comp Neurol 398:179-205.

Cave CB, Squire LR (1992) Intact verbal and nonverbal short-term memory following damage to the human hippocampus. Hippocampus 2:151-163.

Courtney SM, Ungerleider LG, Keil K, Haxby JV (1997) Transient and sustained activity in a distributed neural system for human working memory. Nature 386:608-611.

Davachi L (2006) Item, context and relational episodic encoding in humans. Curr Opin Neurobiol 16:693-700.

Davachi L, Mitchell JP, Wagner AD (2003) Multiple routes to memory: distinct medial temporal lobe processes build item and source memories. Proc Natl Acad Sci U S A 100:2157-2162.

Deadwyler SA, Hampson RE (2004) Differential but complementary mnemonic functions of the hippocampus and subiculum. Neuron 42:465-476.

Diana RA, Yonelinas AP, Ranganath C (2007) Imaging recollection and familiarity in the medial temporal lobe: a three-component model. Trends Cogn Sci 11:379-386.

Dobbins IG, Wagner AD (2005) Domain-general and domain-sensitive prefrontal mechanisms for recollecting events and detecting novelty. Cereb Cortex 15:1768-1778.

Duncan K, Curtis C, Davachi L (2009) Distinct memory signatures in the hippocampus: intentional states distinguish match and mismatch enhancement signals. J Neurosci 29:131-139.

Eacott MJ, Gaffan D, Murray EA (1994) Preserved recognition memory for small sets, and impaired stimulus identification for large sets, following rhinal cortex ablations in monkeys. Eur J Neurosci 6:1466-1478.

Egorov AV, Hamam BN, Fransén E, Hasselmo ME, Alonso AA (2002) Graded persistent activity in entorhinal cortex neurons. Nature 420:173-178.

Eichenbaum H, Yonelinas AP, Ranganath C (2007) The medial temporal lobe and recognition memory. Annu Rev Neurosci 30:123-152.

Eldridge LL, Engel SA, Zeineh MM, Bookheimer SY, Knowlton BJ (2005) A dissociation of encoding and retrieval processes in the human hippocampus. J Neurosci 25:3280-3286.

Epstein R, Kanwisher N (1998) A cortical representation of the local visual environment. Nature 392:598-601.

Epstein RA, DeYoe EA, Press DZ, Rosen AC, Kanwisher N (2001) Neuropsychological evidence for a topographical learning mechanism in parahippocampal cortex. Cogn Neuropsychol 18:481-508.

Fransen E, Alonso AA, Hasselmo ME (2002) Simulations of the role of the muscarinic-activated calcium-sensitive nonspecific cation current INCM in entorhinal neuronal activity during delayed matching tasks. J Neurosci 22:1081-1097.

Freedman DJ, Riesenhuber M, Poggio T, Miller EK (2001) Categorical rep- 
resentation of visual stimuli in the primate prefrontal cortex. Science 291:312-316.

Fuster JM (2001) The prefrontal cortex-an update: time is of the essence. Neuron 30:319-333.

Gaffan D, Murray EA (1992) Monkeys (Macaca fascicularis) with rhinal cortex ablations succeed in object discrimination learning despite 24-hr intertrial intervals and fail at matching to sample despite double sample presentations. Behav Neurosci 106:30-38.

Gazzaley A, Rissman J, Desposito M (2004) Functional connectivity during working memory maintenance. Cogn Affect Behav Neurosci 4:580-599.

Glover GH, Lai S (1998) Self-navigated spiral fMRI: interleaved versus single-shot. Magn Reson Med 39:361-368.

Glover GH, Law CS (2001) Spiral-in/out BOLD fMRI for increased SNR and reduced susceptibility artifacts. Magn Reson Med 46:515-522.

Hannula DE, Ranganath C (2008) Medial temporal lobe activity predicts successful relational memory binding. J Neurosci 28:116-124.

Hannula DE, Tranel D, Cohen NJ (2006) The long and the short of it: relational memory impairments in amnesia, even at short lags. J Neurosci 26:8352-8359.

Harrison SA, Tong F (2009) Decoding reveals the contents of visual working memory in early visual areas. Nature 458:632-635.

Hartley T, Bird CM, Chan D, Cipolotti L, Husain M, Vargha-Khadem F, Burgess N (2007) The hippocampus is required for short-term topographical memory in humans. Hippocampus 17:34-48.

Hasselmo ME, Stern CE (2006) Mechanisms underlying working memory for novel information. Trends Cogn Sci 10:487-493.

Haxby JV, Petit L, Ungerleider LG, Courtney SM (2000) Distinguishing the functional roles of multiple regions in distributed neural systems for visual working memory. Neuroimage 11:145-156.

Henson RN, Rugg MD, Shallice T, Josephs O, Dolan RJ (1999) Recollection and familiarity in recognition memory: an event-related functional magnetic resonance imaging study. J Neurosci 19:3962-3972.

Insausti R, Juottonen K, Soininen H, Insausti AM, Partanen K, Vainio P, Laakso MP, Pitkänen A (1998) MR volumetric analysis of the human entorhinal, perirhinal, and temporopolar cortices. AJNR Am J Neuroradiol 19:659-671.

Jiang Y, Haxby JV, Martin A, Ungerleider LG, Parasuraman R (2000) Complementary neural mechanisms for tracking items in human working memory. Science 287:643-646.

Jonides J, Lewis RL, Nee DE, Lustig CA, Berman MG, Moore KS (2008) The mind and brain of short-term memory. Annu Rev Psychol 59:193-224.

Kim DH, Adalsteinsson E, Glover GH, Spielman DM (2002) Regularized higher-order in vivo shimming. Magn Reson Med 48:715-722.

Kirchhoff BA, Wagner AD, Maril A, Stern CE (2000) Prefrontal-temporal circuitry for episodic encoding and subsequent memory. J Neurosci 20:6173-6180.

Kirwan CB, Jones CK, Miller MI, Stark CE (2007) High-resolution fMRI investigation of the medial temporal lobe. Hum Brain Mapp 28:959-966.

Lehky SR, Tanaka K (2007) Enhancement of object representations in primate perirhinal cortex during a visual working-memory task. J Neurophysiol 97:1298-1310.

Litman L, Awipi T, Davachi L (2009) Category-specificity in the human medial temporal lobe cortex. Hippocampus 19:308-319.

Meunier M, Bachevalier J, Mishkin M, Murray EA (1993) Effects on visual recognition of combined and separate ablations of the entorhinal and perirhinal cortex in rhesus monkeys. J Neurosci 13:5418-5432.

Meunier M, Hadfield W, Bachevalier J, Murray EA (1996) Effects of rhinal cortex lesions combined with hippocampectomy on visual recognition memory in rhesus monkeys. J Neurophysiol 75:1190-1205.

Murray EA, Mishkin M (1998) Object recognition and location memory in monkeys with excitotoxic lesions of the amygdala and hippocampus. J Neurosci 18:6568-6582.

Nichols EA, Kao YC, Verfaellie M, Gabrieli JD (2006) Working memory and long-term memory for faces: evidence from fMRI and global amnesia for involvement of the medial temporal lobes. Hippocampus 16:604-616.

O'Kane G, Insler RZ, Wagner AD (2005) Conceptual and perceptual novelty effects in human medial temporal cortex. Hippocampus 15:326-332.

Olson IR, Page K, Moore KS, Chatterjee A, Verfaellie M (2006a) Working memory for conjunctions relies on the medial temporal lobe. J Neurosci 26:4596-4601.

Olson IR, Moore KS, Stark M, Chatterjee A (2006b) Visual working mem- ory is impaired when the medial temporal lobe is damaged. J Cogn Neurosci 18:1087-1097.

Otto T, Eichenbaum H (1992) Complementary roles of the orbital prefrontal cortex and the perirhinal-entorhinal cortices in an odor-guided delayed-nonmatching-to-sample task. Behav Neurosci 106:762-775.

Paller KA, Wagner AD (2002) Observing the transformation of experience into memory. Trends Cogn Sci 6:93-102.

Pelli DG (1997) The VideoToolbox software for visual psychophysics: transforming numbers into movies. Spat Vis 10:437-442.

Pfeuffer J, Van de Moortele PF, Ugurbil K, Hu X, Glover GH (2002) Correction of physiologically induced global off-resonance effects in dynamic echo-planar and spiral functional imaging. Magn Reson Med 47:344-353.

Piekema C, Kessels RP, Mars RB, Petersson KM, Fernández G (2006) The right hippocampus participates in short-term memory maintenance of object-location associations. Neuroimage 33:374-382.

Piekema C, Fernández G, Postma A, Hendriks MP, Wester AJ, Kessels RP (2007) Spatial and non-spatial contextual working memory in patients with diencephalic or hippocampal dysfunction. Brain Res 1172:103-109.

Postle BR (2006) Working memory as an emergent property of the mind and brain. Neuroscience 139:23-38.

Preston AR, Wagner AD (2007) The medial temporal lobe and memory. In: Neurobiology of learning and memory, Ed 2 (Kesner RP, Martinez JL, eds), pp 305-337. Oxford: Elsevier.

Preston AR, Thomason ME, Ochsner KN, Cooper JC, Glover GH (2004) Comparison of spiral-in/out and spiral-out BOLD fMRI at 1.5 and $3 \mathrm{~T}$. Neuroimage 21:291-301.

Preston AR, Bornstein AM, Hutchinson JB, Gaare ME, Glover GH, Wagner AD (2009) High-resolution fMRI of content-sensitive subsequent memory responses in human medial temporal lobe. J Cogn Neurosci. Advance online publication. Retrieved September 1, 2009. doi:10.1162/jocn.2009.21195.

Pruessner JC, Li LM, Serles W, Pruessner M, Collins DL, Kabani N, Lupien S, Evans AC (2000) Volumetry of hippocampus and amygdala with highresolution MRI and three-dimensional analysis software: minimizing the discrepancies between laboratories. Cereb Cortex 10:433-442.

Pruessner JC, Köhler S, Crane J, Pruessner M, Lord C, Byrne A, Kabani N, Collins DL, Evans AC (2002) Volumetry of temporopolar, perirhinal, entorhinal and parahippocampal cortex from high-resolution MR images: considering the variability of the collateral sulcus. Cereb Cortex 12:1342-1353.

Quintana J, Fuster JM (1999) From perception to action: temporal integrative functions of prefrontal and parietal neurons. Cereb Cortex 9:213-221.

Ranganath C, Blumenfeld RS (2005) Doubts about double dissociations between short- and long-term memory. Trends Cogn Sci 9:374-380.

Ranganath C, D'Esposito M (2001) Medial temporal lobe activity associated with active maintenance of novel information. Neuron 31:865-873.

Ranganath C, Yonelinas AP, Cohen MX, Dy CJ, Tom SM, D’Esposito M (2004) Dissociable correlates of recollection and familiarity within the medial temporal lobes. Neuropsychologia 42:2-13.

Ranganath C, Cohen MX, Brozinsky CJ (2005) Working memory maintenance contributes to long-term memory formation: neural and behavioral evidence. J Cogn Neurosci 17:994-1010.

Rissman J, Gazzaley A, D’Esposito M (2008) Dynamic adjustments in prefrontal, hippocampal, and inferior temporal interactions with increasing visual working memory load. Cereb Cortex 18:1618-1629.

Schon K, Hasselmo ME, Lopresti ML, Tricarico MD, Stern CE (2004) Persistence of parahippocampal representation in the absence of stimulus input enhances long-term encoding: a functional magnetic resonance imaging study of subsequent memory after a delayed match-to-sample task. J Neurosci 24:11088-11097.

Serences JT, Ester EF, Vogel EK, Awh E (2009) Stimulus-specific delay activity in human primary visual cortex. Psychol Sci 20:207-214.

Shrager Y, Gold JJ, Hopkins RO, Squire LR (2006) Intact visual perception in memory-impaired patients with medial temporal lobe lesions. J Neurosci 26:2235-2240.

Shrager Y, Levy DA, Hopkins RO, Squire LR (2008) Working memory and the organization of brain systems. J Neurosci 28:4818-4822.

Small SA (2002) The longitudinal axis of the hippocampal formation: its anatomy, circuitry, and role in cognitive function. Rev Neurosci 13:183-194. 
Squire LR (1992) Memory and the hippocampus: a synthesis from findings with rats, monkeys, and humans. Psychol Rev 99:195-231.

Stark CE, Okado Y (2003) Making memories without trying: medial temporal lobe activity associated with incidental memory formation during recognition. J Neurosci 23:6748-6753.

Stern CE, Corkin S, González RG, Guimaraes AR, Baker JR, Jennings PJ, Carr CA, Sugiura RM, Vedantham V, Rosen BR (1996) The hippocampal formation participates in novel picture encoding: evidence from functional magnetic resonance imaging. Proc Natl Acad Sci U S A 93:8660-8665.

Suzuki WA (2009) Comparative analysis of the cortical afferents, intrinsic projections and interconnections of the parahippocampal region in monkeys and rats. In: The cognitive neurosciences, Ed 4 (Gazzaniga MS, ed), pp 659-674. Cambridge, MA: MIT.

Suzuki WA, Amaral DG (1994a) Topographic organization of the reciprocal connections between the monkey entorhinal cortex and the perirhinal and parahippocampal cortices. J Neurosci 14:1856-1877.

Suzuki WA, Amaral DG (1994b) Perirhinal and parahippocampal cortices of the macaque monkey: cortical afferents. J Comp Neurol 350:497-533.

Suzuki WA, Zola-Morgan S, Squire LR, Amaral DG (1993) Lesions of the perirhinal and parahippocampal cortices in the monkey produce longlasting memory impairment in the visual and tactual modalities. J Neurosci 13:2430-2451.

Suzuki WA, Miller EK, Desimone R (1997) Object and place memory in the macaque entorhinal cortex. J Neurophysiol 78:1062-1081.

Taylor KJ, Henson RN, Graham KS (2007) Recognition memory for faces and scenes in amnesia: dissociable roles of medial temporal lobe structures. Neuropsychologia 45:2428-2438.

Turchi J, Saunders RC, Mishkin M (2005) Effects of cholinergic deafferentation of the rhinal cortex on visual recognition memory in monkeys. Proc Natl Acad Sci U S A 102:2158-2161.
Van Cauter T, Poucet B, Save E (2008) Delay-dependent involvement of the rat entorhinal cortex in habituation to a novel environment. Neurobiol Learn Mem 90:192-199.

Wagner AD, Schacter DL, Rotte M, Koutstaal W, Maril A, Dale AM, Rosen BR, Buckner RL (1998) Building memories: remembering and forgetting of verbal experiences as predicted by brain activity. Science 281:11881191.

Warrington EK, Taylor AM (1973) Immediate memory for faces: long- or short-term memory? Q J Exp Psychol 25:316-322.

Witter MP, Amaral DG (1991) Entorhinal cortex of the monkey: V. Projections to the dentate gyrus, hippocampus, and subicular complex. J Comp Neurol 307:437-459.

Witter MP, Van Hoesen GW, Amaral DG (1989) Topographical organization of the entorhinal projection to the dentate gyrus of the monkey. J Neurosci 9:216-228.

Yassa MA, Stark CE (2009) A quantitative evaluation of cross-participant registration techniques for MRI studies of the medial temporal lobe. Neuroimage 44:319-327.

Young BJ, Otto T, Fox GD, Eichenbaum H (1997) Memory representation within the parahippocampal region. J Neurosci 17:5183-5195.

Zarahn E, Rakitin B, Abela D, Flynn J, Stern Y (2005) Positive evidence against human hippocampal involvement in working memory maintenance of familiar stimuli. Cereb Cortex 15:303-316.

Zeineh MM, Engel SA, Bookheimer SY (2000) Application of cortical unfolding techniques to functional MRI of the human hippocampal region. Neuroimage 11:668-683.

Zeineh MM, Engel SA, Thompson PM, Bookheimer SY (2003) Dynamics of the hippocampus during encoding and retrieval of face-name pairs. Science 299:577-580. 